\title{
MIGRACIONES EN EL RIO AMAZONAS EN LAS ULTIMAS DECADAS, SECTOR CONFLUENCIA RIOS UCAYALI Y MARAÑON - ISLA DE IQUITOS
}

\author{
E eva Tuukki* \\ Päivi J okinen* \\ R isto Kalliola**
}

\section{RESUMEN}

En el presente trabajo se estudiaron los cambios en el cauce del río A mazonas, usando material de percepción remota desde el año 1956. También se estudiaron fluctuaciones en el nivel del río durante 60 años. Se realizaron mediciones de formaciones erosionadas y sedimentadas, los cambios en su totalidad en el área estudiada y la variación dentro del área.

Los resultados presentan una subida del nivel del agua desde los años setenta y el aumento en las cantidades de las formaciones erosionadas y sedimentadas. Sin embargo, por los defectos en las fuentes de información y en la toma de datos, es necesario interpretar los resultados cautel osamente.

Se han distinguido cuatro tipos de secciones en el sector estudiado del río A mazonas según su morfología y dinámica fluvial. Las secciones con varios cauces (trenzado, anastomosado) son siempre separadas por secciones con un solo cauce (directo, meándrico). Cada sección ha permanecido, en términos generales, invariable en los últimos 40 años. En base a la división se puede inferir el comportamiento futuro del río en las próximas décadas. Sin embargo, los cambios imprevisibles del río son una parte de su suceso normal; y por eso, es muy difícil el control del mismo.

Palabras C laves: Dinámica fluvial, niveles de inundación, percepción remota, Perú, Río A mazonas.

Departamento de Biología, Universidad de Turku, 20014 T urun yliopisto, Finlandia. Fax: + 358213335564

Departamento de Geografía, Universidad de Turku, 20014 Turun yliopisto, Finlandia. Fax: + 358213335869 


\section{ABST RACT}

$N$ atural changes of the river A mazon were studied using multitemporal remote sensing imagery since 1956 and data on water level fluctuations during 60 years. Deposition and erosion patches were measured with the purpose to investigate changes in river morphology and their spatial variations within the study area.

The results show that the flood level has been arising from the 1970's on, and also the rates of erosion and deposition have been increasing. However, because of defects on the data, caution must be exercised while interpreting these observations.

Four types of sections were distinguished in the river A mazon, representing different morphological characters and features of fluvial dynamics. Multichannelled sections (braided or anastomosing) are always separated by a section with one single channel (straight or meandering). Each one of these sections has maintained its character throughout the monitored period of 40 years. A lthough it may be possible to predict the behavior of the A mazon river for the nearest future, the dynamic nature of this river makes it very difficult to anticipate changes in a longer run.

Key Words: Fluvial dynamics, flooding, remote sensing, Peru, A mazon river.

\section{INTRODUCCION}

Todos los que viven en Iquitos o lo visitan, conocen el problema grave de la ciudad. Los puertos y las orillas del río están siendo erosionados o rellenados por los sedimentos transportados por el río A mazonas.

El problema de Iquitos es una parte de un proceso normal de la dinámica fluvial: la migración del río.

La migración del río Amazonas en el noreste peruano es permitida por la topografía casi plana de la llanura amazónica. Fuerzas hidráulicas, fluctuaciones en el volumen y velocidad del agua y procesos de erosión y deposición provocan 
la migración del río. Los procesos fluviales crean meandros en el río; la erosión se concentra al lado cóncavo y la deposición al lado convexo de los meandros.

Los meandros son una expresión del patrón del curso del río. Los factores que determinan un patrón de curso son la cantidad y el carácter de carga de sedimentos traídos por los ríos, y su descarga.

Los ríos pueden clasificarse de acuerdo al patrón de sus cursos (Reineck y Singh, 1980; M orisawa, 1985): Ios ríos de curso directo y meándrico presentan un solo cauce, mientras que los ríos de cursos trenzados y anastomosados contienen divisiones sucesivas y rejuntamientos de los canales alrededor de las islas aluviales. En ríos anastomosados las islas son más estables que en las de cursos trenzados.

La dinámica fluvial tiene un efecto extenso sobre el medio ambiente abiótico y biótico en la amazonía peruana (Kalliola et al., 1987; Kalliola et al. , 1992a y 1992b). Durante las inundaciones, grandes cantidades de agua penetran en las planicies de inundación y, los ríos de agua blanca, como el Amazonas, traen consigo una carga considerable de sedimentos fértiles, con origen en los Andes (Sioli, 1984; Furch y Klinge, 1989; Rodríguez et al., 1992; Kalliola et al., 1993; Linna 1993). y, además de eso, la mayor parte de los sedimentos superficiales en áreas no inundables es también de origen fluvial (Räsänen et al., 1987).

L a migración del río A mazonas, el río más largo y caudaloso del mundo, es uno de los hechos más citados de la amazonía.

El comportamiento caprichoso del A mazonas ya fue registrado en el año 1761 por el misionero jesuita San Joaquin de Omaguas que viajó por el A mazonas (Uriarte, 1986). En el libro "El río que se aleja de García (1987) se han acumulado las fuentes históricas del comportamiento del A mazonas cerca de la ciudad de Iquitos.

Posteriormente, se han realizado varios estudios de la migración del río A mazonas frente a la ciudad de Iquitos para intentar detener la erosión que afecta los puertos de la misma (entre otros: M cCreary-Koretsky-Engineers (MKE), 1965; Repetto, 1991; y Observaciones del Servicio de Hidrografía y Navegación de la A mazonía (SHNA)). García \& Bernex de Falen (1994) han expandido los estudios cerca de Iquitos para concernir las interrelaciones entre la dinámica fluvial, la vegetación y el crecimiento poblacional durante los últimos veinte años. Kalliola et al. (1992b) han realizado un estudio sobre la migración de los 
ríos U cayali, M arañón y A mazonas entre los años 1979 y 1983, y la distribución de la vegetación en los ríos de diferentes patrones.

A demás de los anteriores, se han realizado otros estudios científicos sobre el movimiento del río A mazonas en Brasil (Sternberg, 1960; M ertes 1985), y de los ríos U cayali y M arañón en el Perú (Dumont et al., 1990).

En el presente estudio, se utilizan material extenso de percepción remota de casi 40 años y datos de los cambios en el nivel del río de 60 años. Para interpretar extensamente el comportamiento del río, el estudio cubre una sección total de $140 \mathrm{~km}$ de longitud del A mazonas. Con ayuda de los antecedentes históricos del río se intenta buscar una tendencia en base a la que se puedan predecir los movimientos del mismo.

A demás, este estudio prepara una base para las investigaciones posteriores sobre la influencia de la dinámica fluvial en la vegetación en las islas del río A mazonas. L os resultados concernientes con ecología vegetal, se publicarán en el futuro.

\section{MATERIALES Y METODOS}

\subsection{A rea de estudio}

Los ríos U cayali y Marañón se juntan para formar el río A mazonas en el Noreste peruano. El área de estudio, unos $140 \mathrm{~km}$ de longitud, se extiende desde la confluencia de los ríos Marañón y U cayali hasta la Isla de Iquitos (Figura 1).

El río A mazonas presenta un patrón anastomosado (Puhakka et al., 1992). El nivel del río fluctúa en función a la época de creciente, que se presenta en los meses de diciembre a mayo, y a la época de vaciante en los meses de junio a octubre.

En este trabajo para mayor claridad se muestran ubicaciones a lo largo del río, así como las distancias desde la confluencia de los ríos U cayali y $M$ arañón ( $p$. ej. Ia ciudad de Iquitos está situada en el km 118, distancia medida desde la confluencia de los ríos ya mencionados). 


\subsection{Niveles del río}

Se estudiaron fluctuaciones en el nivel del río A mazonas desde el año 1933 hasta el año 1995. Los datos del nivel del río son de las siguientes fuentes, a saber:

- años 1933 - 1961: Dirección de Administración Portuaria del M inisterio de Hacienda (según Hydrotecnic Corporation, 1962).

- años 1962 - 1967: ONERN (1976).

- años 1968 - 1995: SHNA.

\subsection{Cambios en el cauce del río}

Con material de percepción remota se estudiaron la variación temporal y espacial de los cambios del río A mazonas (Cuadro 1).

Cuadro № 1. Material de percepción remota usado en los análisis de la dinámica fluvial del A mazonas.

\begin{tabular}{|c|c|c|c|c|c|}
\hline Año & Fecha & $\begin{array}{r}\text { Nivel del río } \\
(\mathrm{m} . \mathrm{s}, \mathrm{n}, \mathrm{m})\end{array}$ & $\begin{array}{l}\text { E scala } \\
\text { original }\end{array}$ & M aterial & $\begin{array}{c}\text { Hoja/ } \\
\text { Proyecto }\end{array}$ \\
\hline 1956 & Desconocida & Desconocido & $1: 100000$ & $\begin{array}{l}\text { Fotografía aérea } \\
\text { (Instituto G eográfico } \\
\text { Nacional 1989) }\end{array}$ & 8-p, 9-p \\
\hline 1972 & $\begin{array}{l}\text { junio } 16 \text { - } \\
\text { octubre } 1\end{array}$ & $116-113$ & $1: 150000$ & $\begin{array}{l}\text { M osaico de } \\
\text { fotografía aérea }\end{array}$ & $\begin{array}{l}\text { SAN } \\
214-72-A\end{array}$ \\
\hline 1972 & $\begin{array}{l}\text { junio } 16 \text { - } \\
\text { octubre } 1\end{array}$ & $116-113$ & $1: 20000$ & $\begin{array}{l}\text { F otografía aérea } \\
\text { vertical }\end{array}$ & $\begin{array}{l}\text { SAN } \\
214-215-72\end{array}$ \\
\hline 1972 & Desconocida & Desconocido & $1: 125000$ & $\begin{array}{l}\text { Imágenes de radar } \\
\text { (SLAR) }\end{array}$ & $13-1-D$ \\
\hline 1983 & setiembre 19 & 111 & $1: 250000$ & $\begin{array}{l}\text { M apa planimétrico } \\
\text { (LANDSAT MSS) }\end{array}$ & IFG 1984 \\
\hline 1993 & agosto 5 & 112 & $1: 250000$ & LANDSAT TM & WRS 006.63 \\
\hline
\end{tabular}


Todos los materiales a diferentes escalas se convirtieron a la escala de 1:150000. Se eligió el mapa planimétrico de imágenes de satélite del año 1983 para la comparación con los otros mapas, ya que éste representa un mapa más confiable. Cada imagen se comparó con este mapa usando los sitios con características distintivas, como criterios para su confirmación. Después los cauces de los años 1956, 1972, 1983 y 1993 se dibujaron en transparencias. Se compararon los cauces por parejas, sobreponiendo dibujos de los años sucesivos, y se prepararon mapas de cambios (manchas de deposición y erosión) presentando tres períodos: 1956 - 72 (16 años), 1972 - 83 (11 años) y 1983 - 1993 (10 años).

Los mapas de cambios del río se usaron para investigar 1) los cambios en su totalidad en el área de estudio y 2) las variaciones en estos dentro del área de estudio.

1) Se midieron las superficies de las manchas erosionadas y depositadas de cada período usando un tablero digitalizador. Se contaron cantidades totales de los cambios separadamente para las orillas del río y para las islas.

2) Se examinaron la ubicación y variación de los procesos erosivos y de sedimentación en el cauce del río con un análisis de las líneas de transectas. En el medio del canal del río se estimó una línea mediana, y en cada dos kilómetros líneas transversales a la misma. A lo largo de las líneas transversales se midieron longitudes de formaciones de erosión y de deposición. Se estudiaron en total 193 transectos representando tres períodos.

\section{RESULTADOS}

\subsection{Niveles del río}

Durante el período 1933 - 1970, los mínimos y máximos anuales del río se mantienen relativamente por debajo de los valores promedio (Figura 2). Solamente en algunos años el máximo o el mínimo suben sobre los promedios de todos los años. 
En los años setenta, el nivel llega a los valores más altos, y se mantiene arriba todo el decenio. Evidenciándose, en líneas generales, que los niveles anuales del río presentan una tendencia ascendente desde el año 1933 hasta el presente. Sin embargo, las diferencias entre los mínimos y máximos anuales (amplitud) varían entre cinco y diez metros en todo el período de 60 años.

\subsection{Cambios en el cauce del río}

L a inestabilidad es muy pronunciada en ciertas secciones del río, p. ej. en los primeros kilómetros del río A mazonas ( $\mathrm{km} 0$ - 15) y entre los km 55 - 75 (Figuras 3 y 4). En estas etapas la formación de las islas es muy reciente; éstas desaparecen en unos 10 años y otras nuevas están desarrollándose en el canal.

Ciertas áreas del río parecen ser susceptibles a cambios de un tipo durante un período y a cambios contrarios en otro período. En las islas al oeste de la Isla M uyuy ( $\mathrm{km} \mathrm{90),} \mathrm{tanto} \mathrm{la} \mathrm{erosión} \mathrm{como} \mathrm{deposición} \mathrm{han} \mathrm{afectado} \mathrm{fuertemente} \mathrm{el}$ paisaje aluvial durante períodos sucesivos. En consecuencia, los sedimentos de los diferentes períodos forman un mosaico.

L a migración unidireccional del río no es común en el área estudiada. Sólo en el km 110 se puede observar una deposición continua en cada período en el lado convexo del cauce del río. Debido a que la orilla cóncava externa, por su parte, es objeto de erosión, el cauce está moviéndose hacia el Noreste.

En el meandro entre los km 40 - 55, el lado convexo ha sufrido erosión durante el primer período, pero solamente deposición posteriormente. En el lado externo del meandro los cambios son contrarios.

Aunque la mayoría de las secciones estudiadas sufren cambios frecuentemente, existen al gunos lugares sin cambios distinguibles durante casi 40 años.

N ormalmente las orillas de las islas grandes son susceptibles a cambios; pero las orillas en la parte sur (margen derecha del río Amazonas) de la Isla Panguana ( $\mathrm{km} 85$ - 100) son excepcionalmente estables. Las secciones estables más largas de un solo cauce (cercanías del km 20 y el km 80) tienen unos diez kilómetros de longitud, son estrechas y sin formación de islas. 
Tanto la cantidad de formaciones erosionadas como la de formaciones sedimentadas ha aumentado en el área de estudio (Cuadro 2). Existen diferencias entre los cambios en las islas y las orillas.

Durante las dos últimas décadas, las islas vienen siendo más susceptibles a la deposición que a la erosión salvo en el área de la isla de Iquitos (Cuadro 3). En general, las islas sufren más deposición que las orillas, y las orillas más erosión que las islas.

C uadro № 2. Superficies totales y anuales de erosión y deposición en el área de estudio en los períodos estudiados.

Período

Años

Erosión E rosión

Deposición

Deposición

anual (ha)

total (ha) anual (ha) total (ha)

Cuadro № 3. Superficies anuales de erosión y deposición en las orillas y en las islas del Río Amazonas.

$\begin{array}{llllll}1956-72 & 16 & 5042 & 315 & 4440 & 278 \\ 1972-83 & 11 & 6551 & 596 & 7440 & 676 \\ 1983-93 & 10 & 6892 & 689 & 7422 & 742\end{array}$

\begin{tabular}{lll}
\hline Período & Orillas del río & Islas
\end{tabular}

\begin{tabular}{ccccc}
\cline { 2 - 4 } & $\begin{array}{c}\text { Erosión } \\
\text { anual (ha) }\end{array}$ & $\begin{array}{c}\text { Deposición } \\
\text { anual (ha) }\end{array}$ & $\begin{array}{c}\text { Erosión } \\
\text { anual (ha) }\end{array}$ & $\begin{array}{c}\text { Deposición } \\
\text { anual (ha) }\end{array}$ \\
\hline $1956-72$ & 148 & 120 & 167 & 157 \\
$1972-83$ & 303 & 247 & 293 & 429 \\
$1983-93$ & 420 & 348 & 270 & 394 \\
\hline
\end{tabular}




\section{DISCUSION}

\subsection{Niveles del río}

Gentry y López; (1980) han sostenido, en base a los datos de los niveles del río A mazonas en Iquitos desde el año 1962 hasta el año 1978, que el nivel de inundación y la diferencia entre el mínimo y el máximo anual (amplitud) han aumentado. Repetto (1991) por su parte, evidencia que los niveles en la misma área en los años 1970 y 1980 registran valores más altos que el promedio del total de los años registrados desde 1935.

También nuestro análisis parece mostrar que la fluctuación en el nivel del río tiene una tendencia a presentar valores más altos durante la última parte del período estudiado. Sin embargo, a diferencia del estudio de Gentry y López-Parodi, la fluctuación en las amplitudes es aleatoria. Por cierto, la variación en las fuentes de información y posiblemente, en la manera de medir los niveles del río, puede deformar los resultados. Por ejemplo, el nivel del agua subió en el año 1968 cuando también cambió la fuente de los datos.

Todos los estudios no están de acuerdo en lo que respecta a que los niveles del río A mazonas han aumentado. El estudio de Gentry y López-Parodi ha encontrado crítica en base a sus parámetros metodológicos y estadísticos (Nordin \& M eade, 1982; Sternberg, 1987). En los estudios del río A mazonas en $M$ anaus, ni se encontró una tendencia segura en el nivel (Sternberg, 1987) ni en el caudal del río (Richey et al., 1989) en un período de unos 80 años y, por las razones arriba mencionadas, es preciso mantener ciertas dudas en la tendencia de incremento en el análisis.

\subsection{Cambios en el cauce del río}

El estudio muestra cómo los cambios en el cauce del río varían tanto espacialmente (a lo largo del río mismo) como temporalmente (períodos de cambios o de estabilidad en los mismos sitios, entre años diferentes).

A demás, parece que los procesos de erosión y deposición han aumentado en los últimos períodos estudiados.

Sin embargo, este análisis puede tener inexactitudes por tres razones mayores: los materiales de percepción remota fueron obtenidos en diferentes fechas con 
diferentes niveles de agua, los períodos estudiados son de diferentes duraciones, y la interpretación del material de percepción remota fue difícil. Por ejemplo, en el período 1972 - 1983 la diferencia entre los niveles de agua es $\quad 2$ - $5 \mathrm{~m}$. Por estas razones es posible que este resultado refleje aspectos de metodología y no cambios reales en el río.

Especialmente en las islas es pronunciada la situación irregular de sedimentos de diferentes períodos. Esta característica es típica para los ríos de curso anastomosado; en contraste, a lo largo de los cursos meándricos se puede observar migración unidireccional de curvas (K alliola et al., 1992b). También un río anastomosado, como el río A mazonas, puede incluir partes meándricas, donde las orillas pueden mostrar tendencias hacia la erosión o la deposición. Cuando esta última es continua, un complejo de orillares será formado como consecuencia de una migración unidireccional del río.

Los cambios más extensos se encuentran en secciones anchas donde la velocidad de la corriente disminuye y la carga de sedimentos tiene la posibilidad de acumularse y formar islas.

Según Cornejo \& Riva (1992), las islas del río A mazonas presentan una relativa estabilidad pero "en los últimos años se ha notado que los procesos erosivos son más acentuados que los de acumulación". Según nuestro estudio, una mayor deposición ocurrió en las islas durante el último período estudiado (1983 - 1993). Particularmente las islas pequeñas son susceptibles de cambios, pero también las más grandes sufren cambios continuamente.

En unas secciones los cambios están concentrados en el cauce, afectando principalmente las islas fluviales, y en otras en las márgenes del río. Por otro lado, en algunas secciones ambos fenómenos se pueden presentar conjuntamente. Por ejemplo, la deposición en las islas puede formar un impedimento para el flujo y la presión de la corriente se acentúa sobre las orillas del río.

A pesar de la inestabilidad temporalmente continua en la mayoría del río A mazonas, también existen lugares casi estables. En muchos de estos lugares el espacio para los movimientos del río está limitado por el margen de la planicie de inundación. En la mayor parte, estos lugares estables están caracterizados por un canal estrecho.

Para entender mejor los cambios en el río, es necesario recordar que normalmente, no solamente un factor sino que varios factores en conjunto 
están afectando su dinámica. A demás de los fenómenos presentados en este estudio, ocurren otros tipos de cambios, y la información sobre éstos provendrá de estudios de geomorfología de la planicie de inundación. Por ejemplo la anchura de la planicie de inundación (que llega a ser hasta de 40 $\mathrm{km}$ ) indica inestabilidad pronunciada y migraciones de mucha mayor escala, las que pueden incluir mecanismos que no han ocurrido durante los últimos 40 años. Por ejemplo la Isla de Iquitos ha sido cortada por el río en el año 1994, siendo uno de los cambios más notorios en el río desde el año 1948.

\subsection{Secciones del río}

En el río A mazonas se pueden distinguir secciones distintas concernientes a su comportamiento y morfología. A unque generalmente el río presenta un curso anastomosado, también existen cursos de otros tipos.

A continuación, las características de las secciones del río se han clasificado en cuatro tipos (Figura 3 y Cuadro 4).

\section{Cuadro № 4. Características de cuatro tipos de secciones en los primeros 140 kilómetros del río A mazonas (véase Figura 3).}

\begin{tabular}{lllll}
\hline & Directo & Meándrico & Trenzado & A nastomosado \\
\hline Anchura del cauce & Estrecha & Estrecha & Bastante ancha & A ncha \\
Islas & No & No & Pequeñas, fugaces & $\begin{array}{l}\text { Grandes, } \\
\text { permanentes }\end{array}$ \\
Cambios & No & Unidireccionales & En mosaicos & En mosaicos \\
Secciones & 2 y 6 & 4 y 8 & 1 y 5 & 3,7 y 9 \\
\hline
\end{tabular}


Excluyendo a la sección de la Isla de Iquitos, el río puede ser distinguido en dos partes en las cuales los tipos se repiten en el mismo orden: trenzado $(T)$, directo (D), anastomosado (A) y meándrico (M). Las secciones trenzadas 0 anastomosadas son siempre separadas por secciones con un solo cauce: directo 0 meándrico. Todas estas secciones han permanecido básicamente iguales durante los casi 40 años estudiados.

Basados en esta división se puede inferir el comportamiento del río en un futuro próximo, quizás en décadas; pero la predicción para un tiempo más largo es incierta debido a mecanismos a mayor escala, como el tectonismo o los cambios climáticos.

Sin embargo, aunque en algunos lugares el río causa destrucción, la deposición de material de alta fertilidad en otros, es un factor importante que puede ser de una gran potencialidad para el desarrollo de la zona.

\section{CONCLUSIONES}

1) El nivel de inundación del río A mazonas viene subiendo desde la primera mitad de los años setenta. Pero, por los inexactitudes en los datos, esto posiblemente sea falso.

2) Las superficies de las formaciones erosionadas y sedimentadas parecen haber aumentado.

3) Los cambios son continuos en unos sitios; pero en otros, los cambios son muy variables.

4) Se pueden distinguir cuatro tipos de secciones en el río A mazonas: sección de curso directo, meándrico, trenzado y anastomosado. Las cuales se repiten ordenadamente y en conjunto a lo largo del curso estudiado exceptuando la sección en la Isla de Iquitos.

5) Para entender la conducta del río, se necesita tener en cuenta no únicamente una parte corta del río sino también otras secciones más distantes.

6) Los cambios del río A mazonas son una parte del suceso normal de los ríos. Por eso, es muy difícil el tratar de controlarlo, y es mejor acomodarse a sus resultados aprovechando sus aspectos positivos. 


\section{AGRADE CIMIE NTO}

Este trabajo ha sido realizado en el marco del Proyecto Amazonía de la Universidad de Turku (PAUT). Expresamos nuestra gratitud a Abel Sarmiento Tulumba y A ntonio Layche Gómez por su colaboración en el campo, y a Kalle Ruokolainen y Luisa Rebata Hernani por sus sugerencias críticas. Ofrecemos nuestro reconocimiento a Carlos Calle Barco (IIAP) por su valiosa colaboración y al Servicio de Hidrografía y Navegación de la A mazonía (SHNA), especialmente a J orge Paredes Bravo, por los datos de los niveles del río. El trabajo ha tenido el apoyo de la A cademia de Finlandia, la Sociedad de la U niversidad de Turku, la Sociedad de T. y J. Wallden y la U nión Europea (STD 3). 


\section{BIBLIOGRAFIA}

MCCREARY - KORETSKY - ENGINEERS 1965. Iquitos riverbank protection. Second part of Phase II of studies for Riverbank Protection along the Amazon River at Iquitos, Peru. Lima, Perú.

DUMONT, J. F., LAM OTTE, S. \& KAHN, F. 1990. Wetland and upland forest ecosystems in Peruvian A mazonia: Plant species diversity in the light of some geological and botanical evidence. For. Ecol. Manag. 33/34: pp. $125-139$.

CORNEJO, H. \& RIVA, R. 1992. Estudio de suelos y capacidad de uso mayor de las tierras zona Tamshiyacu-Indiana. IIAP, Iquitos, Perú. 51 p.

FURCH, K. \& KLINGE, H. 1989. Chemical relationships between vegetation, soil and water in contrasting inundation areas of A mazonia. En: J. Proctor (ed.): Mineral nutrients in tropical forest and savanna ecosystems, pp. 189 - 204. Special publication number 9 of the British ecological society. Blackwell Scientific Publications, Cambridge.

GARCIA, J. 1987. El río que se aleja: cambio del curso del Amazonas, historia y estudio técnico. CETA, Iquitos, Perú. 57 p.

GARCIA, J. \& BERNEX DE FALEN, N. 1994. El río que se aleja: cambio del curso del Amazonas, estudio histórico-técnico. CETA-IIAP, Iquitos, Perú. Segunda edición. 51 p.

GENTRY, A.H. \& LOPEZ-PARODI, J. 1980. Deforestation and Increased Flooding of the U pper A mazon. Science 210: pp. 1354 - 1356.

HYDROTECNIC CORPORATION 1962. Defensa de la Ribera del Río Amazonas en Iquitos.

InStituto GeOgRAFICO nACIONAL 1989. Atlas del Perú. Lima, Perú. pp. $68-69$.

KALLIOLA, R. SALO, J. \& M äKINEN, Y. 1987. Regeneración Natural de las Selvas en la A mazonía Peruana 1: Dinámica fluvial y sucesión ribereña. 
Memorias del M useo de Historia Natural J avier Prado NN18. U niversidad N acional M ayor de San M arcos (U N M SM ). Lima, Perú. 102 p.

KALLIOLA, R., SALO, J., PUHAKKA, M.\& RAJASILTA, M. 1992a. New site formation and colonizing vegetation in primary succession on the western A mazon floodplains. J. E col. 79: pp. 877 - 901.

KALLIOLA, R., SALO, J., PUHAKKA, M.\& RAJASILTA, M., HÄME, T., NELLER, R.J., RÄSÄNEN, M.E. \& DANJOY ARIAS, W.A. 1992b. Upper A mazon channel migration. Implications for vegetation perturbance and succession using bitemporal Landsat MSS images. Naturwiss. 79: pp. 75 - 79.

KALLIOLA, R., LINNA, A., PUHAKKA, M., SALO, J. \& RÄSÄNEN, M. 1993. $M$ ineral nutrients in fluvial sediments from the Peruvian A mazon. Catena 20: pp. 333 - 349.

LINNA, A. 1993: Factores que contribuyen a las características del sedimento superficial en la selva baja de la A mazonía peruana. En: Kalliola, R., Puhakka, M. \& Danjoy, W. (eds.): A mazonía Peruana. Vegetación húmeda tropical en el Ilano subandino. pp. 87 - 97.

MERTES, L.A.K. 1985. Floodplain Development and Sediment Transport in the Solimoes-A mazon River, Brazil. Tesis de maestria, Universidad de W ashington, Estados U nidos. 108 p.

MORISAWA, M. 1985. Rivers. Form and process. New Y ork, Estados U nidos. $222 \mathrm{p}$.

NORDIN, C.F. \& MEADE, R.H. 1982. Deforestation and Increased Flooding of the Upper A mazon. Science 215: pp. 426 - 427.

ONERN 1976. Inventario, Evaluación e Integración de los Recursos Naturales de la Selva: Zona Iquitos, Nauta, Requena y Colonia Angamos. Lima, Perú. 269 p. y 6 mapas.

PUHAKKA, M., KALLIOLA, R., SALO J. \& RAJASILTA, M. 1992. River types, site evolution and successional vegetational pattern in Peruvian A mazonia. J. Biogeogr. 19: pp. 651 - 665. 
REINECK H.E. \& SINGH, I. B. 1980. Depositional sedimentary environments with reference to terrigenous clastics. Segunda edición. Springer-V erlag, Berlin. $549 \mathrm{p}$.

REPETTO M. S. A. 1991. Estudio de protección de la ribera del T.F. de Iquitos. Empresa $\mathrm{N}$ acional de Puertos S. A. Perú.

RICHEY, J.E., NOBRE, C. \& DESER, C. 1989. A mazon River Discharge and Climate Variability: 1903 to 1985. Science 246: pp. 101 - 103.

RODRIGUEZ, F., PAREDES, G., BAZAN, R. \& RAMIREZ, J. 1992. Algunas propiedades físicas, químicas y mineralógicas de materiales sedimentarios recientes en un complejo de orillares del río A mazonas. Folia A mazónica 4: pp. 5 - 26.

RÄSÄNEN, M., SALO, J. \& KALLIOLA, R. 1987. Fluvial perturbance in the Western Amazon Basin: regulation by long-term Sub-Andean tectonics. Science 238: pp. 1398 - 1401.

SALO, J., KALLIOLA, R., HÄKKINEN, I., MÄKINEN, Y., NIEMELÄ, P., PUHAKKA, M. \& COLEY, P.D. 1986. River dynamics and the diversity of A mazon lowland forest. Nature 322: pp. 254 - 258.

SIOLI, H. 1984. The A mazon and its main affluents: hydrography, morphology of the river courses, and river types. En: Sioli, H. (ed.), The Amazon. Limnology and landscape ecology of mighty tropical river and its basin. Dr. W. J unk Publishers, the Hague. pp. 127 - 165.

STERNBERG，H. 1960. Radiocarbon dating as applied to a problem of A mazonian morphology. Comtes Rendus 18 Congrès International de Géographie. Comité N ational du Brésil, U GI. Rio de J aneiro, Brasil. pp. $399-424$.

STERNBERG, H. 1987. Aggravation of floods in the Amazon river as a consequence of deforestation?. Geografiska Ann. 69A: pp. 201 - 219.

URIARTE, P.M.J. 1986. Diario de un misionero de Maynas. Monumenta Amazónica 1986, Iquitos, Perú. 686 p. 
Figura 1. Ubicación del área de estudio, río Amazonas y su planicie de inundación, en el año 1993. La imagen a la izquierda presenta la fluctuación anual en el nivel del río Amazonas, basada en los datos desde el año 1933 hasta el año 1995. La extensión entre valores de maximum y minimum está oscurecida y el promedio se expone por la línea sólida.
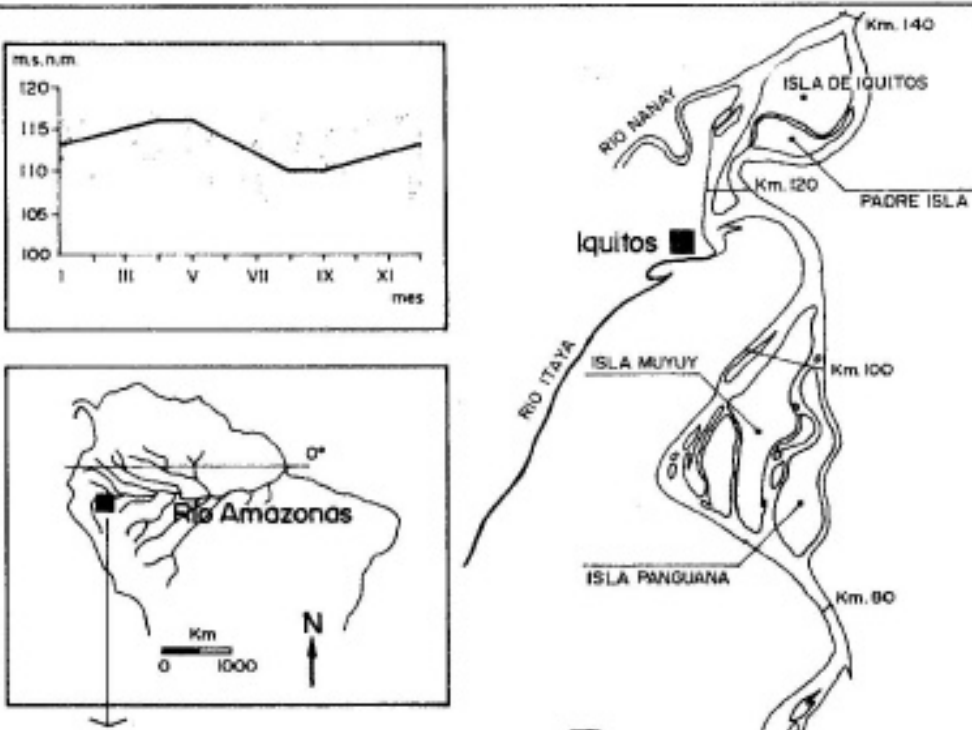

ISLA MUnY

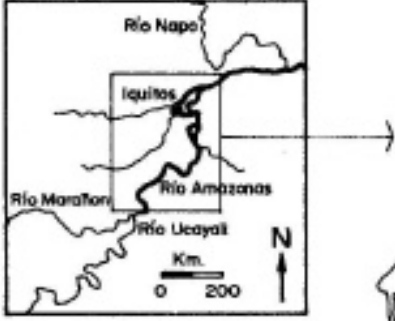

ISLA YACAPANA
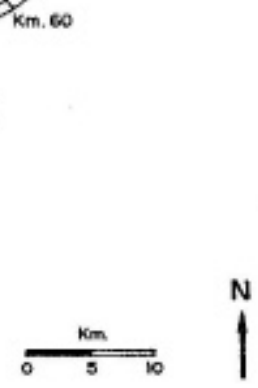
Figura 2. M ínimos y máximos anuales del nivel del río A mazonas en Iquitos desde el año 1933 hasta el año 1995 (arriba), y amplitudes anuales (abajo) del nivel del río. Las áreas oscurecidas indican años cuando el nivel o las amplitudes eran más altos que los promedios. Se carece de datos de los mínimos entre los años 1962 y 1967. Las fechas de los materiales de percepción remota, usados en este estudio, están marcados con puntos.

m.s.n.m.

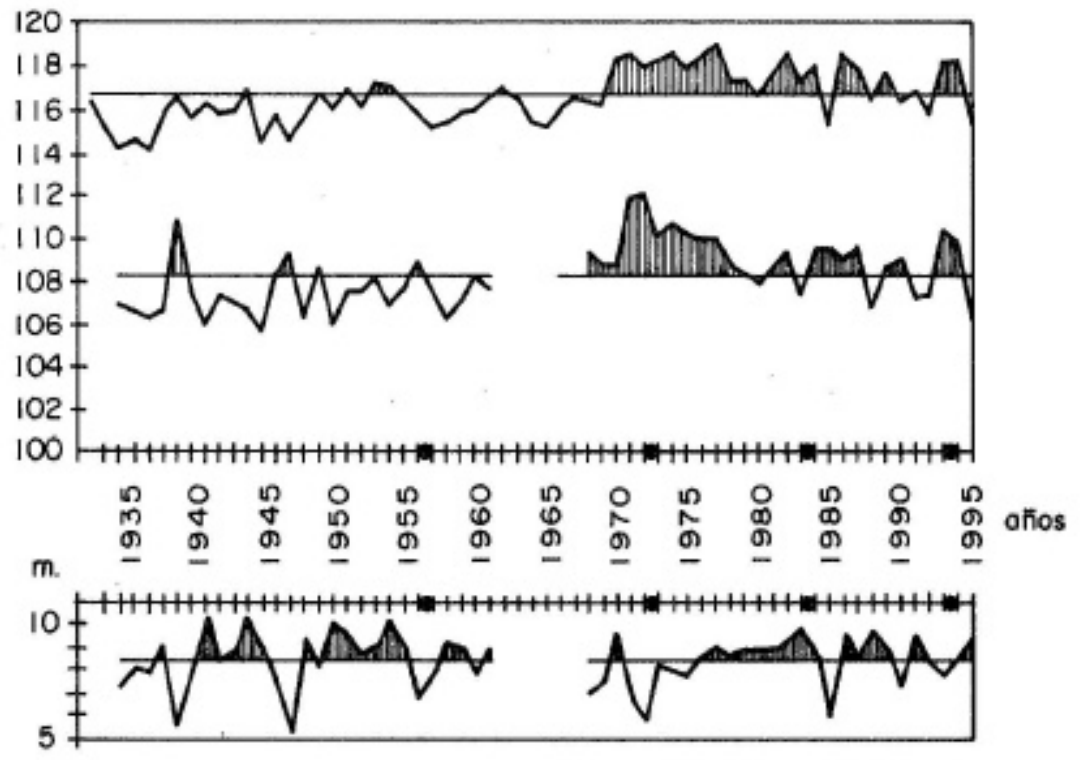


Figura 3. Las islas y las orillas del río Amazonas se forman en terrenos de diferentes edades. L a oscuridad de las unidades palidece hacía los áreas más antiguas. Con una línea de puntos se ha marcado la parte faltante de la fotografía aérea tomada en el año 1956. En la imagen del año 1993 se han distinguido secciones representando cuatro tipos de curso: directo (D), meándrico (M), trenzado (T) y anastomosado (A).

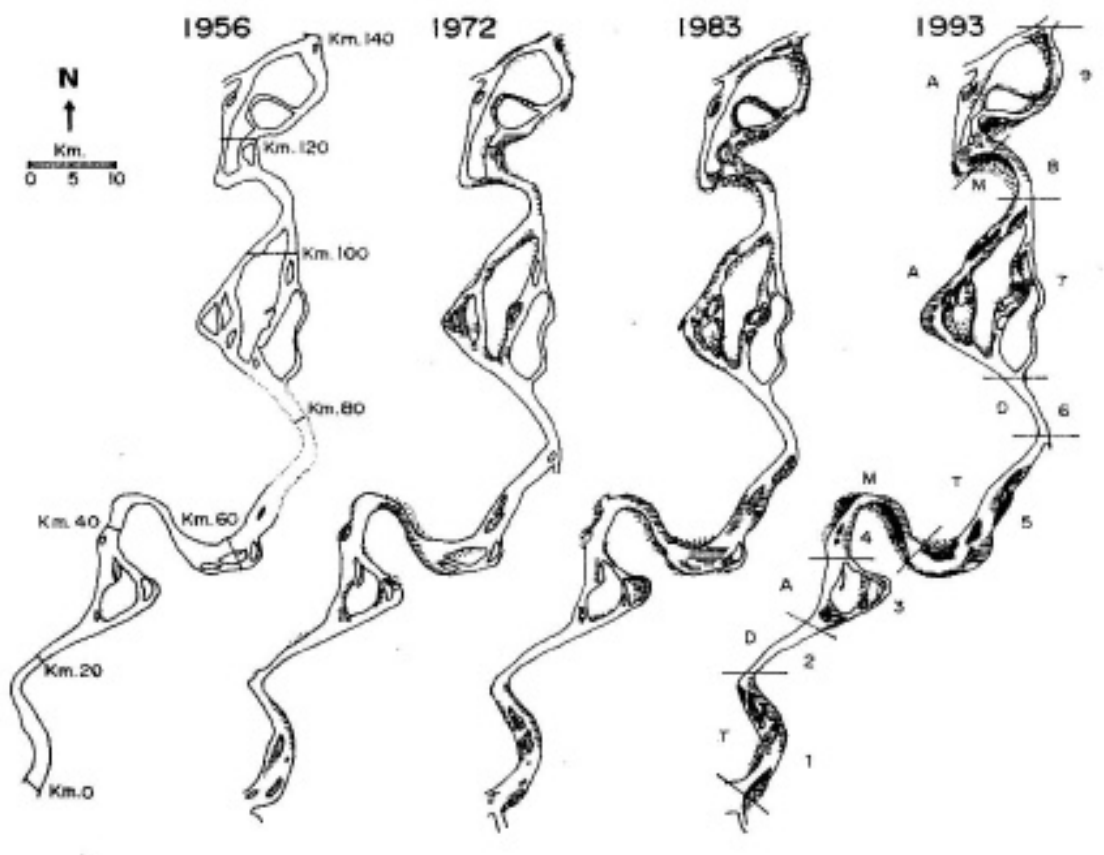

Artea que se ariginan antes de, y burente

los periobse estudiados

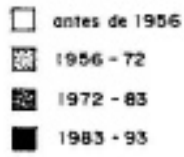

A tosión fes les ûlt imos 10 ahos 1 
Figura 4. Anchuras de las manchas de erosión y deposición total del canal del río Amazonas entre los años estudiados, a lo largo de las líneas transversales.
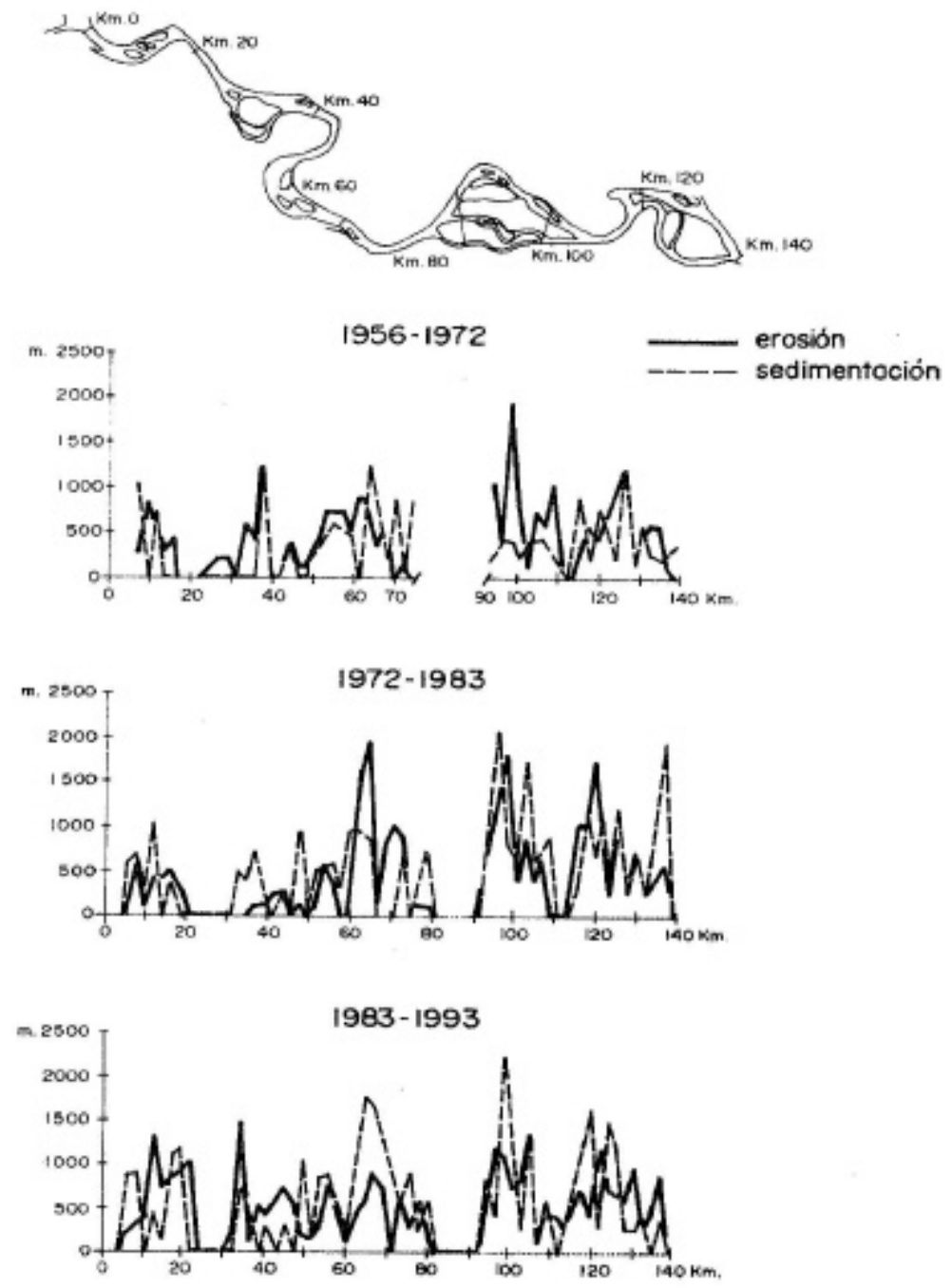